\title{
Research for High-speed Real-time Acquisition and Storage System in Engineering Flight Simulation Platform
}

\author{
Zhongping Jiang ${ }^{1, a}$, Hong $\mathrm{Xu}^{1, \mathrm{~b}}$, Tao $\mathrm{Liu}^{2, \mathrm{c}}$ \\ ${ }^{1}$ School of Computer Science, Beihang University, Beijing 10086, China \\ ${ }^{2}$ School of Biological and Medical Engineering, Beihang University, Beijing 10086, China \\ abuaa0300@163.com, bh@buaa.edu.cn, ctao.liu@buaa.edu.cn
}

Keywords: engineering flight simulation platform, data collection and storage, data model, build algorithm of data block, cache queue.

\begin{abstract}
Engineering flight simulation platform is an important equipment in research and development of the new aircraft. The high-speed and real-time collection and storage system is one of the important subsystem in engineering flight simulation platform. In order to solve two key problems, which are general and real time, in the process of system development. This paper gives the common data model the different structure, which adapts to the simulation different users of platform; proposes the high-speed and real-time acquisition algorithm, which is based on cache queue, guarantees millisecond collection and storage of system.
\end{abstract}

\section{Introduction}

Engineering flight simulation platform has played an important role in the aerospace and aviation. It provides a good experimental environment for studying and designing the new aircraft ${ }^{[1]}$. In order to guarantee the collection data, which was produced in the stage of the research or test, can reflect accurately the aircraft parts, aircraft subsystem, and even the whole performance of the aircraft, it must be real-time acquisition. Through processing and analyzing these data, it not only evaluates the properties and functions of the experimental objects, but also provides the important reference for the research and performance optimization of the equipment.

According to the large number of simulation data in the engineering flight simulation experiment, this paper adopts a high-speed and real-time acquisition and storage method. Section 2 studies the common data model of simulation variables, and data storage blocks construction method in the data acquisition, and a selective algorithm about real-time high-speed data acquisition and storage, and an algorithm about real-time high-speed data acquisition storage, which is based on cache queue. Section 3 introduces the implementation of the system. Section 4 summarizes the conclusion of this system.

\section{System Research}

\section{General data model of system.}

In the process of simulation experiment, the different simulation computer nodes produce the simulation data by calculating simulation model in real time. Then the data acquisition computers read simulation data from reflective memory in real time, and store in hard disk medium with the test requirements. After the experiment, the data processing computers will process these data by the test requirement. The data acquisition and storage process schematic diagram is shown in Figure 1:

In the simulation experiment, different computer nodes of simulation model produces data and write to the reflective memory network, and then according to requirements of the experiments, the system collect the real-time data by the data acquisition computer, store the data in the disk medium. After the experiment, the data processing computers deal with the data by the needs of the experiment. 
The engineering flight simulation platform, which is studied by us in this paper, faces different kinds of experimental tasks. They may be the different types of aircraft, or single subsystem or multiple subsystems in the same plane. Because of the different experimental tasks, the type of simulation variables, the combination of various kinds of data, which are collected by system are not the same. The type of simulation variables may be char, short, integer, long, float, double, etc. ${ }^{[1]}$. By the way, the simulation variables are not existing alone, which are depended different subsystems in the experiment, the correlative relationship model between simulation variables and subsystems model is described as follows:

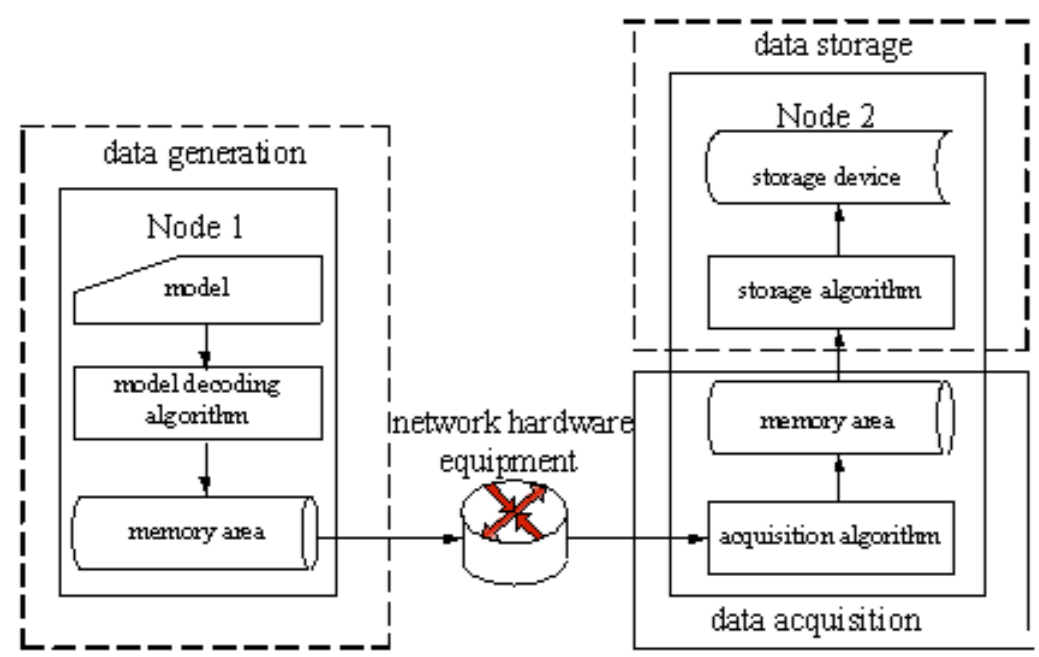

Figure 1. Data collection and Storage process structure

$\mathrm{R}=\{\mathrm{ID}$, varID, sysID, relation $\}$

The ID is the unique identification of each pair of correlation relationship, varID is on behalf of simulation variables, sysID is on behalf of an aircraft subsystem which simulation variables are belonged to, relation is on behalf of the relationship between the variables and the subsystem. It includes associations relationship, the values of varID and sysID are independent of each other.

Data model relation of system is mainly used to describe the various relationship between aircraft subsystem and simulation variables. data model relation of simulation variable shows as follows:

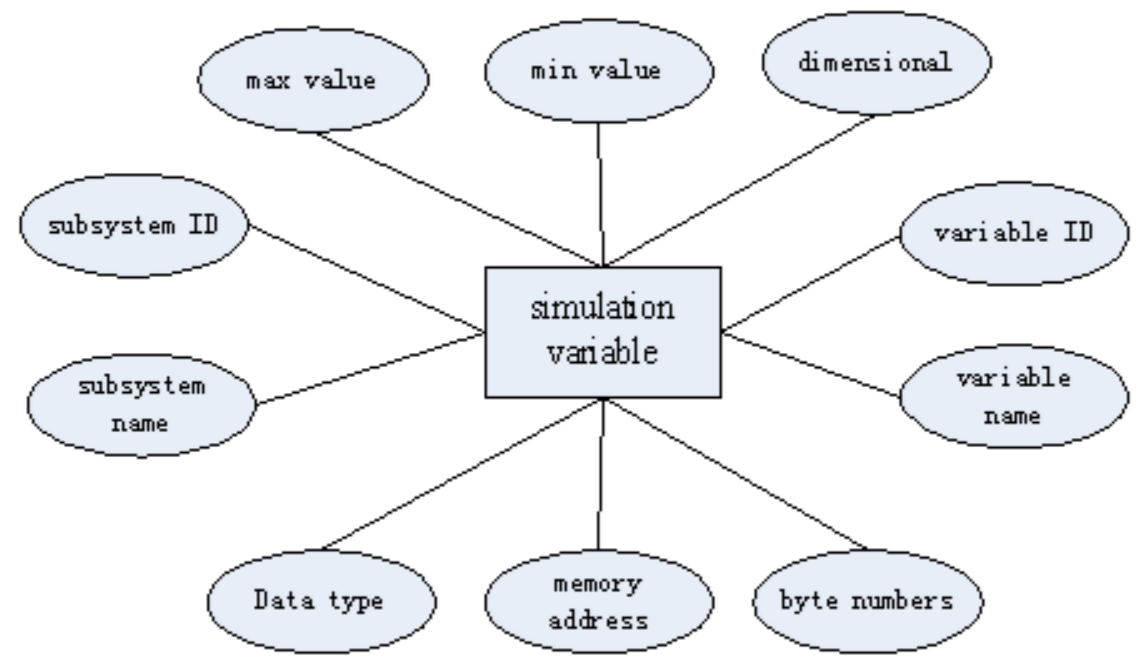

Figure 2. General data model of system

\section{The building of the data storage blocks.}

The building principle of the data storage blocks. The simulation data retains in different reflective memory units. And different types of data use the different number of memory cells in reflective memory. It causes low ability about collecting data from reflective memory network. This is because 
that a reflective memory unit operation requires multistep preparation and follow-up work, which needs to waste time ${ }^{[3]}$. And the collection efficiency is worse and worse by increasing the number of variables.

The study have shown that: If the starting address of the first simulation variable is known, and ith simulation variable occupy ki $(1<=\mathrm{ki}<=4)$ number of storage units. Therefore, starting address of nth simulation variable is:

$$
\mathrm{LOC}(\mathrm{An})=\mathrm{LOC}(\mathrm{A} 1)+\sum_{i=1}^{n-1} k i \text {. }
$$

When the number of simulation variables is fixed, these variables are building too many storage blocks. The number of blocks is B, the acquisition time is T. less blocks take less time. By the way, if $\mathrm{Ti}<\mathrm{Tj}$, then $\mathrm{Bi}<\mathrm{Bj}$.

Figure 3 shows the data acquisition speed of 3000 simulation variables in different blocks.

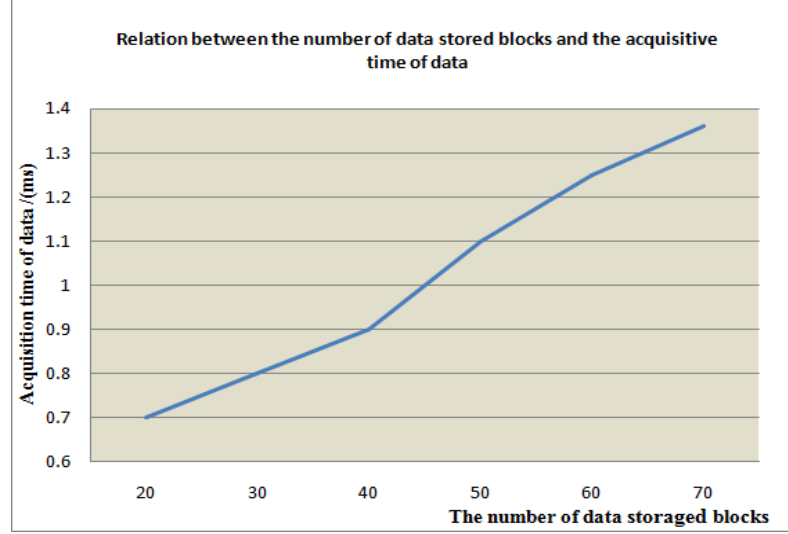

Figure 3. Data acquisition time and different acquisition blocks

However, the physical address of simulation variables in the subsystems in engineering flight simulation platform is associated with the physical parts in the plane, which causes the state of no continuity and complexity. Therefore, the system need to improve the rate of collection and storage by using the appropriate building principle of the data storage blocks, which reduces the number of collection and storage blocks.

The building method of the data Storage blocks. The building methods of the data storage blocks are as follows:

a) Different subsystems build different data storage blocks.

The various subsystems of simulation experiment are provided by the different designing company, which causes the storage location independently, the storage location between each subsystem is discontinuous. So it is not right that make the simulation variable in different subsystems into the same data storage block.

b) All the continuous simulation variables set in each subsystem built into the data storage blocks.

In each subsystem, if the storage location of simulation variables is continuous, but the type of simulation variables are not same, the system make these variables into the same data storage block by shielding the type of difference. If the storage location of simulation variables is discontinuous, it need to pick up the discrete simulation variables and build into the new data storage blocks.

c) Special simulation variables of subsystems process respectively.

In the subsystems, the storage address of some simulation variables is very special, the address is not in the same period. Pick up these special variables alone. If the storage address of these simulation variables is continuous, making into a data storage blocks, if it is discontinuity, make it into the new data storage block alone.

According to the data storage blocks method, the simulation variables built into continuous Storage blocks, which reduces the time of the data acquisition. But different number of data acquisition blocks for influence the time of data acquisition, Therefore, in the system implementation, we need to limit the number of data storage blocks. 


\section{The algorithm of Selective collection and storage.}

The design idea of selective collection and storage: build fewer data storage blocks by reducing the number of acquisition simulation variables, which ensure that the collection and storage period can meet the needs of experiment[1].Algorithm must solve the problems are as follows:

a) adapting to the different the acquisition template.

b) automatic matching between acquisition template and collection data.

c) the management of the acquisition template and collection data.

Selective collection and storage algorithm is described as follows:

a) the user select the simulation variables, and the number of simulation variables is less than 200 , build a acquisition template, according to the number of experimental need.

b) system build the data storage blocks on the basic of the acquisition template.

c) the system begins to collect data when receiving the beginning signal of collection from master station .

d) the system reads data from reflective memory network through the data storage blocks, and then stores in the hard disk.

e) when receiving the stop signal of acquisition, the system stop to collect, and then release the data storage blocks.

Algorithm process as shown in figure 4:

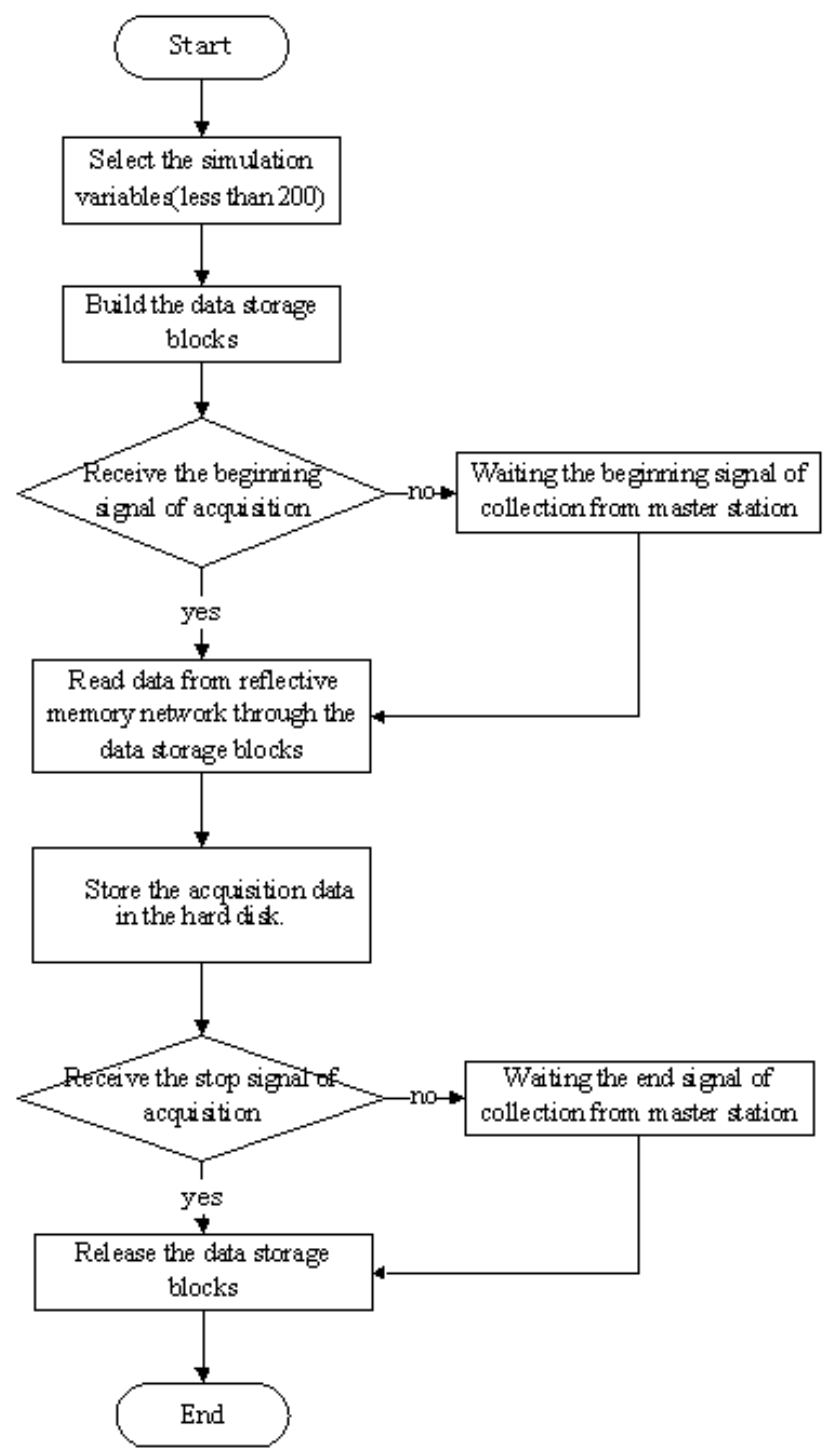

Figure 4. Flow chart of selective collection and storage 
The Selective collection and storage algorithm has reached the requirement of millisecond collection and storage, but since the number of variables is less than 200, which cannot be applied to millisecond acquisition and storage a large number of simulation variable. By the way, the works need to build manually the collection template before the experiment, which increases the workload. Most important, selective collection and storage don't solve the contradiction between the low speed writing and the high speed reading, which limits the efficiency of collection and storage.

The algorithm of collection and storage based on cache queue.

The design idea of collection and storage based on buffer queue is: reduce the contradiction between low-speed writing and high-speed reading by cache queue. In this way, it can make sure millisecond acquisition and storage of a large number of simulation variables. The main problem of the algorithm is that how to use the cache queue to reduce the contradiction between high speed acquisition and storage at low speed.

The description of the algorithm based on cache queue is as follows:

a) The user select all the simulation variables before the experiment.

b) system build the data storage blocks on the basic of the acquisition template and cache queue.

c) the system begins to collect data when receiving the beginning signal of collection from master station .

d) the system reads data from reflective memory network through the data storage blocks, and then stores in the cache queue.

e) system retrieves data from the cache queue, and then stores in hard disk in real time .

f) when receiving the stop signal of acquisition, the system stop to collect, and then release the data storage blocks and cache queue.

Algorithm process as shown in figure 5:

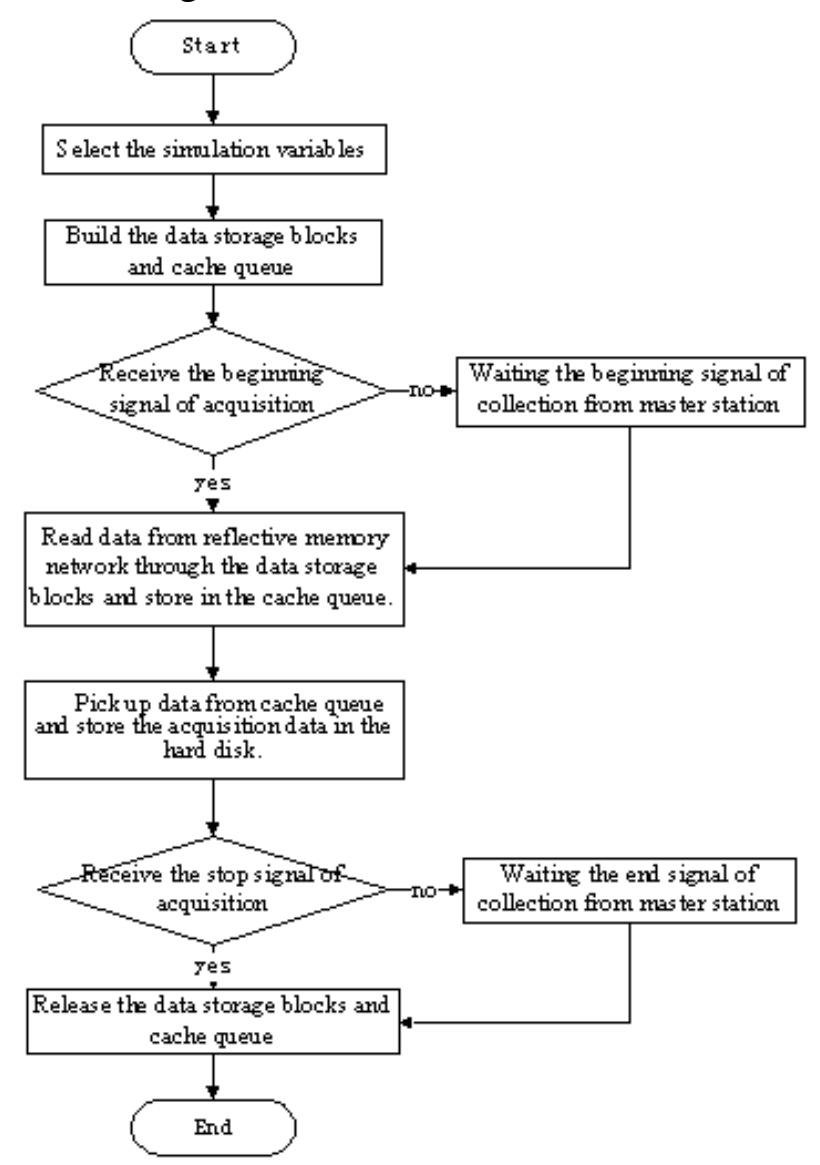

Figure 5. Flow chart of collection and storage based on cache queue

In this algorithm, the cache queue includes cache data control, space management and synchronization mechanism of queue ${ }^{[7,8,9]}$.

I in the cache queue, the data has the following features: 
a) all the data of each subsystem store in one queue node.

b) The state of data in a queue node has three states: initialization, no read and read. When the node initialization, the state of node is initialization; when the node get data of a subsystem, the state of node is initialization no read; when data in node has been written to disk, the state of node is read.

II The space management of queue cache has the following features:

1) The space size of each queue node is initialized to a fixed size before data collection

2) In the process of data collection, if the new data push the queue, but the memory space is not enough, allocates dynamically memory; when the data in the queue is pop, clean up immediately the memory place of the data.

3) After the data collection, save the last set of data in time, and clear the memory place of the cache queue.

III The synchronization mechanism of cache queue adopts the following strategies:

In the process of data collection and storage, reader thread is responsible for reading data from the reflective memory network and saving into the buffer queue, writer thread is responsible for saving the data from the cache queue to the hard disk. Read thread and write thread follow the principle of the synchronization between write and read. The system only has a reader and a writer. The writer thread must wait until the read operation is completed, only when the reader thread obtain a complete subsystem of data, the writer thread can save the next node of buffer queue to the hard disk. The read thread and the write thread share a read-write lock.

\section{System Implementation}

In order to guarantee the millisecond acquisition and storage of a lot of simulation variables. In the implementation of system, this paper uses the data block construction method, reduce the data acquisition time; acquisition and storage algorithm based on cache queue, alleviate the contradiction between high speed acquisition and storage at low speed, improve the efficiency of storage. The structure diagram of data acquisition and storage system is as follows:

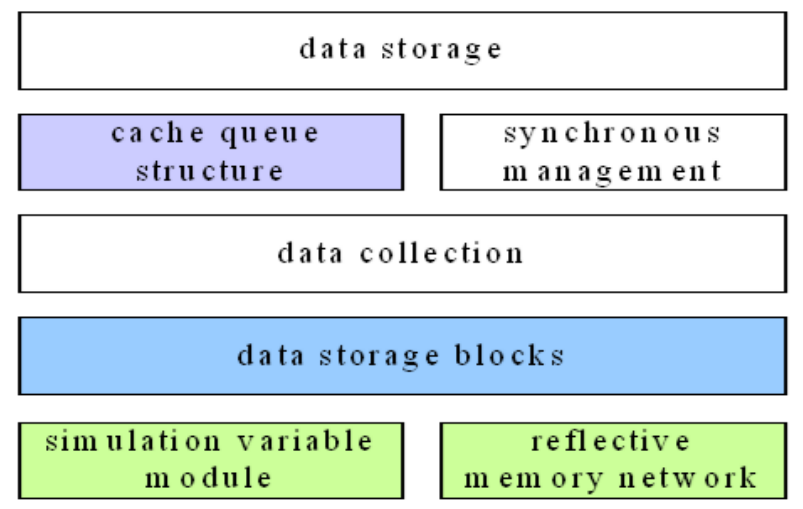

Figure 6. System structure of data acquisition and storage

From the figure 6, the system builds data storage blocks by the simulation variable template before acquisition. In the process of real time data collection, system reads data from the reflective memory network by the data storage blocks and stores in the cache queue, which is based on the principle of first in and first out. And then the system picks up data from the queue and stores in the hard disk. When the acquisition is complete, all the data are written to the hard disk, the cache queue and data storage blocks are released.

With the data collection and storage algorithm based on cache queue, realized the collection and storage of 3000 simulation variables in $2 \mathrm{~ms}$. The 3000 simulation variables are distributed in more than 20 subsystems. The number of simulation variable of each subsystem is not the same, the type is not unified, the memory address of discontinuity between subsystems. The data information of acquisition is as follow: 


\begin{tabular}{|l|c|c|}
\hline Simulation Time & First Variable & Second Variable \\
\hline .003 & 38343241 & 0 \\
\hline .005 & 38343241 & 0 \\
\hline .007 & 38343241 & 0 \\
\hline .009 & 38343241 & 0 \\
\hline .011 & 38343241 & 0 \\
\hline .013 & 38343241 & 0 \\
\hline .015 & 38343241 & 0 \\
\hline .017 & 38343241 & 0 \\
\hline 019 & 38343241 & 0 \\
\hline .021 & 38343241 & 0 \\
\hline .023 & 38343241 & 0 \\
\hline .025 & 38343241 & 0 \\
\hline .027 & 38343241 & 0 \\
\hline .029 & 38343241 & 0 \\
\hline 031 & 38343241 & 0 \\
\hline .033 & 38343241 & 0 \\
\hline
\end{tabular}

Figure 7 part result of collection and store in $2 \mathrm{~ms}$

\section{Summary}

This paper proposes the high-speed and real-time data acquisition and storage methods of system in engineering flight simulation platform, which are the common data model of system, data storage blocks, selective collection and storage algorithm and the collection and storage algorithm based on cache queue. The system has reached the requirement of millisecond collection and storage. The system achieve the high speed acquisition and storage of huge amounts of data.

\section{References}

[1]. YU Ji-chao, SHEN Wei-qun, ZOU Yu-fang. High Speed Real-time Data Acquisition System of Project Aircraft Simulator. Computer Engineering. 2013.

[2]. YU Yue-hua, WU Chao, SHEN Weiqun, SONG Zishan. The development of real-time monitoring system in Engineering flight simulation system. Computer Simulation. 2006.

[3]. Wang Xinmin, Jiang Zhengxiong, Xie Rong, Huang Yu. Design of Distributed Real-Time Flight Control Simulation System Based on VMIC Network, Computer Measurement and Control. 2011.

[4]. LiYi. Design for High speed data acquisition, storage and processing system. Xian university of electronic science and technology. 2009.

[5]. WANG Xuesong. Some key techniques of high-speed data acquisition in the storage device. North Central University. 2013.

[6]. Rongxin, Su Xinyan. The design of data acquisition and processing system based on virtual instrument, North Central University. 2011.

[7]. REN Zhiguo, LI Yanan, ZHANG Longzhong, ZHAO Lilai. Circular queue order storage and algorithm implementation. Journal of gansu union university. 2010.

[8]. Wuqing, Wang huaishan. A Study of Congestion Control Algorithm Based on Active Queue Management. Computer Application Technology. 2008

[9]. Lin Chuan, Wu Jingdong. Improve for a dynamic size of memory management algorithm. Single chip microcomputer and Embedded system Application, Fuzhou University. 2008. 